\title{
Diffusion of Breast Cancer Risk Assessment in Primary Care
}

\author{
Carmen E. Guerra, MD, MSCE, Melani Sherman, BA, and \\ Katrina Armstrong, MD, MSCE
}

Background: Physicians who provide primary care to women have the opportunity to identify patients at high risk for breast cancer who are candidates for risk reduction strategies. Our objective was to determine the prevalence and determinants of the adoption of breast cancer risk assessment by primary care physicians.

Methods: A cross-sectional survey of a nationally representative random sample of 351 internists, family practitioners, and obstetricians-gynecologists. We used a questionnaire that assessed knowledge, attitudes, discussion of breast cancer risk, use of software to calculate breast cancer risk, and ordering of $B R C A 1 / 2$ testing.

Results: Eighty-eight percent of physicians reported discussing breast cancer risk at least once during the previous 12 months; $48 \%$ had ordered or referred a patient for BRCA1/2 testing; and $18 \%$ had used a software program to calculate breast cancer risk. Physicians who had used BRCA1/2 testing or discussed breast cancer risk factors were more likely to be obstetrician-gynecologists and not in a solo practice; the use of risk software was also more common among obstetrician-gynecologists but was also associated with having a family member with breast cancer and a greater knowledge about breast cancer risk. Having patients ask for risk information was associated with the discussion of risk factors but not with the other risk assessment strategies.

Conclusions: Diffusion of breast cancer risk assessment is occurring in primary care practices, with a greater adoption of $B R C A 1 / 2$ testing than of the use of risk assessment software. Adoption of these strategies seems to be related to the salience of breast cancer personally (for the physician) and within the practice, as well as the size of the practice, rather than attitudes about the risk assessment methods. (J Am Board Fam Med 2009;22:272-9.)

In the United States, an estimated 180,510 new cases of breast cancer will be diagnosed and 40,910 women will die of the disease in $2007 .{ }^{1}$ For women

This article was externally peer reviewed.

Submitted 19 July 2008; revised 21 October 2008; accepted 6 November 2008.

From the Department of Medicine, School of Medicine (CEG, MS, KA); the Leonard Davis Institute of Health Economics (CEG, KA); and the Abramson Cancer Center (CEG, KA), University of Pennsylvania, Philadelphia.

Funding: Dr. Guerra acknowledges the National Cancer Institute (Grant K01-CA97925) and the Robert Wood Johnson Foundation Amos Medical Faculty Development Award (Grant 051895). Dr. Armstrong acknowledges the Robert Wood Johnson Foundation Generalist Physician Faculty Scholar Award and the American Cancer Society Research Scholar Grant (Grant 104351).

Conflict of interest: The sponsors did not play a role in the design or conduct of this study or preparation of this manuscript. Drs. Guerra and Armstrong had full access to all of the data in the study and take responsibility for the integrity of the data and the accuracy of the data analysis. None of the authors has a financial conflict of interest related to the subject of the study.

Corresponding author: Katrina Armstrong, MD, MS, University of Pennsylvania, 1204 Blockley Hall, 423 Guardian Drive, Philadelphia, PA 19104 (E-mail: karmstro@mail.med.upenn.edu). at average risk for breast cancer, the lifetime risk of being diagnosed with breast cancer is $13 \% .^{2}$ However, breast cancer risk varies substantially among women, with 5 -year risk ranging from $<0.5 \%$ for a low risk woman in her $40 \mathrm{~s}$ to $>6 \%$ for a high-risk woman in her 70 s. $^{3}$

During the last 10 years, breast cancer risk assessment has become increasingly relevant to physicians who provide primary care to women for several reasons. ${ }^{4}$ The identification of women at high risk for breast cancer has taken on new importance with the Food and Drug Administration's approval of tamoxifen for breast cancer risk reduction in high-risk women in 1998 and the growing data supporting the effectiveness of prophylactic mastectomy among women at very high risk..$^{5-7}$ Since the validation of the Gail model for individual breast cancer risk prediction, ${ }^{8}$ additional software programs for risk prediction have been developed, including the Claus, Couch, ShattuckEidens, Frank, and BRCAPRO models. ${ }^{9-13}$ The 
development of testing for mutations in the major breast cancer susceptibility genes, BRCA1 and $B R C A 2$, has created risk-assessment tools that can both identify women at high risk for breast cancer but also provide information about ovarian cancer risk and cancer risk information for family members. ${ }^{14}$ Finally, evidence continues to link a woman's absolute risk of breast cancer to the risk/benefit ratio of many common decisions, including the use of postmenopausal hormone replacement therapy, the age at which to start mammography screening, and the appropriateness for high-risk screening programs including magnetic resonance imaging. ${ }^{15,16}$

The US Preventive Services Task Force recently issued guidelines for genetic risk assessment and $B R C A 1 / 2$ testing in women with breast and ovarian cancer susceptibility. ${ }^{17}$ These guidelines, given a level B recommendation, state that referral for genetic counseling and consideration for breast cancer screening is recommended for women at increased risk of having BRCA (breast cancer gene) mutations. It is estimated that $2 \%$ of adult women in the general population meet a relatively strict definition of increased risk ( 2 first-degree relatives with breast cancer, one of whom was diagnosed at age 50 or younger; or a combination of 3 or more first- or second-degree relatives with breast cancer; or a combination of both breast and ovarian cancer among first- and second- degree relatives or a firstdegree relative with bilateral breast cancer; or a combination of 2 or more first- or second-degree relatives with ovarian cancer; or a first- or seconddegree relative with both breast and ovarian cancer; or a history of breast cancer in a male relative). ${ }^{15-17}$ However, an additional $5 \%$ to $6 \%$ of women are considered moderate risk for carrying a mutation, where $B R C A 1 / 2$ testing among 1200 women is estimated to prevent 1 case of breast cancer and 3 cases of ovarian cancer. ${ }^{17}$

Despite the recent developments in breast cancer risk assessment, little is known about primary care physicians' use of these new tools for risk assessment or their general discussion of breast cancer risk with their patients. One previous survey of primary care physicians in California investigated the use of tamoxifen and raloxifene and found that $86 \%$ of physicians said they initiate breast cancer risk reduction discussions at least half the time; $45 \%$ had referred a patient for genetic evaluation but did not measure the use of risk calculation software or ordering genetic tests. ${ }^{18,19}$ Thus, the objective of this study was to assess the use of breast cancer risk assessment strategies among primary care physicians and to determine whether the use of $B R C A 1 / 2$ testing and risk prediction software was associated with the provider's personal characteristics, knowledge about breast cancer risk factors, or attitudes about breast cancer risk assessment. We hypothesized that use of these new strategies for risk assessment would be greatest among primary care physicians in whom breast cancer risk was most salient (physicians who had a family history of breast cancer or who saw a greater proportion of female patients) and who had greater knowledge of breast cancer risk factors and more positive attitudes about breast cancer risk assessment.

\section{Methods}

A cross-sectional, nationwide survey of a random sample of 1000 primary care physicians was conducted between June 2002 and June 2004.

\section{Participants}

A random sample of 1000 primary care physicians from obtained from the American Medical Association Masterfile. The sample was stratified by primary specialty as reported in the American Medical Association Masterfile to include an equal number of physicians whose primary practice was internal medicine, obstetrics/gynecology, or family medicine.

\section{Procedures}

The Institutional Review Board of the University of Pennsylvania approved this study. Study particiapants were mailed the questionnaire, a cover letter, and a reply envelope with prepaid postage. In the first mailing, participants were randomized to receive either a handwritten note, a $\$ 5$ incentive, or a handwritten note and a $\$ 5$ incentive. Two subsequent mailings, including the questionnaire, cover letter, and a \$5 incentive, were mailed to nonresponders. Physicians who had not responded to any of the mailings were called and offered a chance to complete the survey by phone or fax or to be mailed another packet if they preferred to complete it as a hard copy.

\section{Survey Instrument}

The PRECEDE model of health behavior (Predisposing, Reinforcing, and Enabling Constructs in Educational Diagnosis and Evaluation), where be- 
haviors are influenced by the presence or absence of predisposing, enabling, and reinforcing factors, was used to guide survey development. ${ }^{20-21}$ The content areas of the questionnaire consisted of 4 sections: (1) physician demographics and practice characteristics; (2) knowledge about breast cancer risk; (3) attitudes about breast cancer risk assessment; and (4) physician behavior related to breast cancer risk assessment. The results of an additional survey content area related to primary care physicians' prescribing of tamoxifen for breast cancer prevention have been previously published. ${ }^{22}$

\section{Independent Variables}

To assess knowledge of breast cancer risk factors, physicians were provided 7 pairs of clinical scenarios about women older than 40 years of age and were asked to identify the woman with the greater risk of developing breast cancer during her lifetime. Knowledge score was the proportion of the 7 cases for which the physician correctly identified the higher-risk case in the pair. To assess attitudes about breast cancer risk assessment, the questionnaire included 5 items assessing beliefs that risk assessment was too time consuming, that risk assessment could increase patient anxiety, that available methods of risk assessment were not accurate enough, that many patients asked for risk information, and that risk information might make low-risk women less likely to adhere to mammography screening. Response scales consisted of a 5-point Likert scale from "strongly agree" to "strongly disagree."

Items adapted from other physician surveys were used to assess sociodemographic and practice characteristics, including the number of primary care physicians in the practice, the average number of patients seen during a week by the physician responding to the survey, medical school affiliation, and the year of graduation from medical school. ${ }^{23}$ We also asked whether any family member had been diagnosed with breast cancer and, if so, which family member.

\section{Outcome Variables}

The questionnaire measured the frequency at which physicians had used any software programs to calculate breast cancer risk, ordered or referred a patient for BRCA1/2 testing, and discussed breast cancer risk factors during the previous 12 months.
Response scales for frequency of use questions were on a 5 -point numerical scale that consisted of 0,1 to 6,7 to 12,12 to 24 , and $>24$ times. Because very few physicians had performed these behaviors multiple times, these responses were collapsed into 0 versus 1 or more.

\section{Statistical Analysis}

STATA SE software (version 8.0, StataCorp, LP, College Station, TX) was used to conduct all the statistical analyses. Descriptive statistics were calculated for physician and practice characteristics. Bivariate statistics using $\chi^{2}$ and $t$ tests were calculated to determine the relationship between physician characteristics, practice characteristics, physician knowledge of and attitudes toward breast cancer risk assessment, and each of the 3 outcome variables (discussion of breast cancer risk factors, use of software program to calculate breast cancer risk, and use of BRCA1/2 testing). Logistic regression was used to adjust the associations between independent variables and the outcome variables (as dichotomous variables) for potential confounding and effect modification. Separate regression models were fit for each of the outcome variables. Covariates were selected based on a priori hypothesis deduced from our theoretical model or on a significant bivariate relationship. Each model adjusted for the known physician demographic and practice characteristics.

\section{Results}

Of the original sample of 1000 physicians, 248 physicians who had incorrect addresses, were no longer practicing, were not a primary care physician, had no female patients, or had died were excluded. Twenty-six physicians refused to participate. Of the remaining 726 questionnaires, 383 surveys were received after all attempts to recover the surveys. After excluding 32 incomplete surveys, a total of 351 questionnaires were available for analysis. The response rate was therefore $48.3 \%$. Responders did not differ significantly from nonresponders in sex, region of the country, specialty, or type of degree (MD vs DO). However, responders had graduated from medical school more recently than nonresponders $(P<.01)$.

The demographic and practice characteristics of the participating physicians are listed in Table 1 . The mean age was 45.6 years and just over two- 


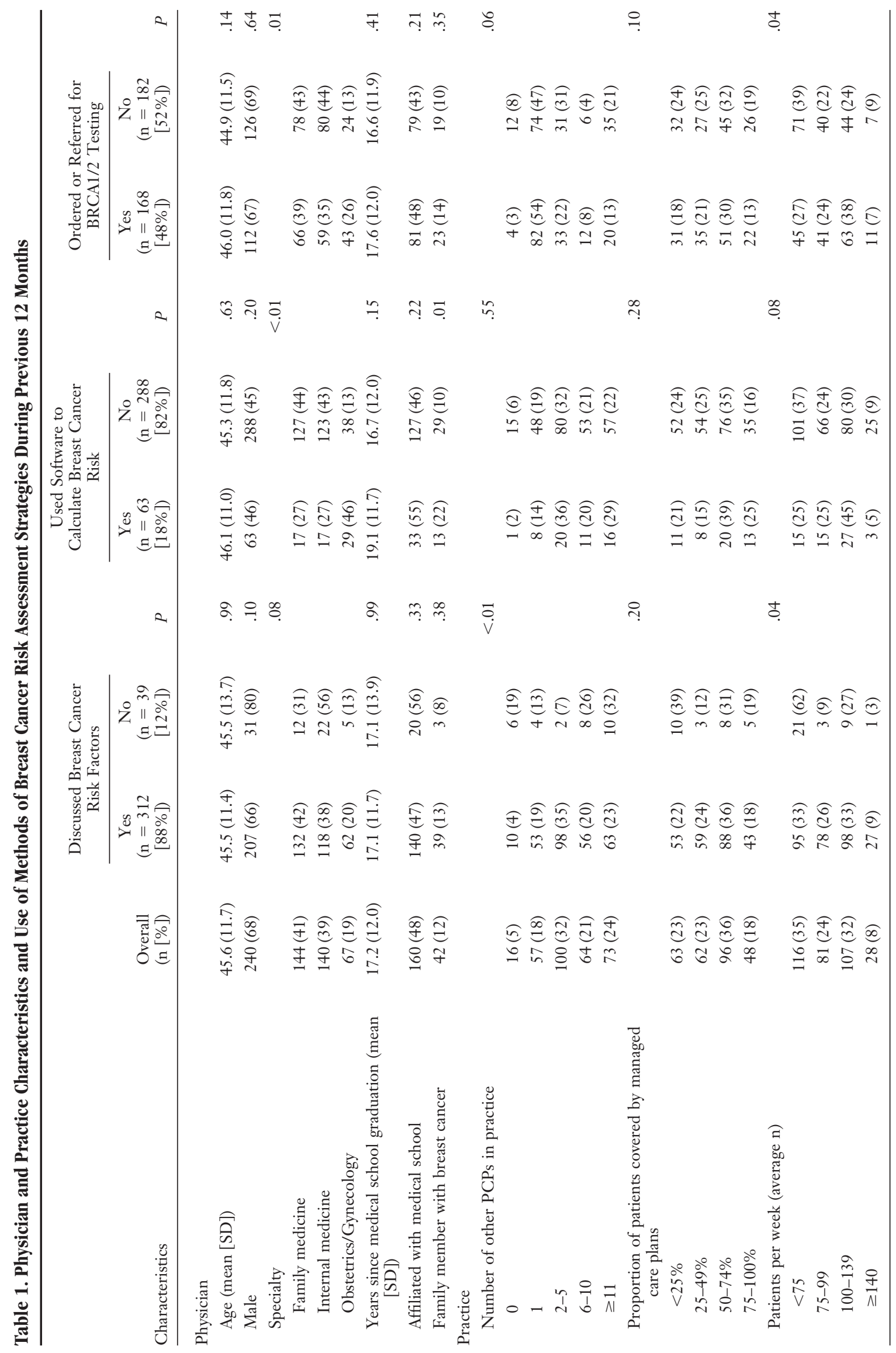

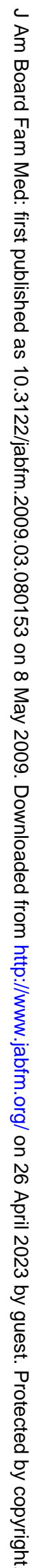


thirds were men. Forty-one percent practiced family practice, $39 \%$ internal medicine, and 19\% obstetrics and gynecology. The mean number of years since graduating from medical school was 17.2. Approximately half had an affiliation with an academic medical center. Twelve percent of physicians had a close family member (parent, sister, spouse, daughter) who was diagnosed with breast cancer. Physician use of methods of breast cancer risk assessment during the previous 12 months revealed that $88 \%$ of physicians had discussed breast cancer risk with a patient within that time $(26 \% 1$ to 6 times, $13 \% 7$ to 12 times, $13 \% 12$ to 24 times, and $37 \%>24$ times); $18 \%$ of physicians had used software to calculate breast cancer risk $(11 \% 1$ to 6 times, $3 \% 7$ to 12 times, $1 \% 12$ to 24 times, and 3\% $>24$ times); and $48 \%$ of physicians had ordered or referred a patient for genetic testing for BRCA1/2 mutations (33\% 1 to 6 times, $8 \% 7$ to 12 times, $4 \%$ 12 to 24 times, and $3 \%>24$ times) during the last 12 months (Table 1). No physicians had used software to calculate breast cancer risk without discussing breast cancer risk and only 5 physicians had ordered or referred for genetic testing without reporting having discussed breast cancer risk factors.

The associations between physician and practice characteristics and breast cancer risk assessment strategies are shown in Table 1. Physician specialty was significantly associated with use of risk software and the use of BRCA1/2 testing, with higher rates among obstetrician-gynecologists than internists or family practitioners. Physician specialty was also correlated with the discussion of risk factors; however, the association did not reach statistical significance. The number of primary care providers in the practice was significantly associated with the discussion of breast cancer risk factors, and there was a trend toward an association with the use of $B R C A 1 / 2$ testing. In addition, having a family member with breast cancer was associated with use of software to calculate risk. Not surprisingly, the use of each of these breast cancer risk assessment strategies increased with the average number of patients seen per week.

Knowledge of breast cancer risk factors was significantly higher among physicians who had used risk assessment software (mean knowledge score for software users, 0.71 vs 0.65 for nonusers; $P<.01$ ), but was not associated with having discussed breast cancer risk or with having ordered genetic testing for BRCA 1/2 mutations (Table 2). The belief that

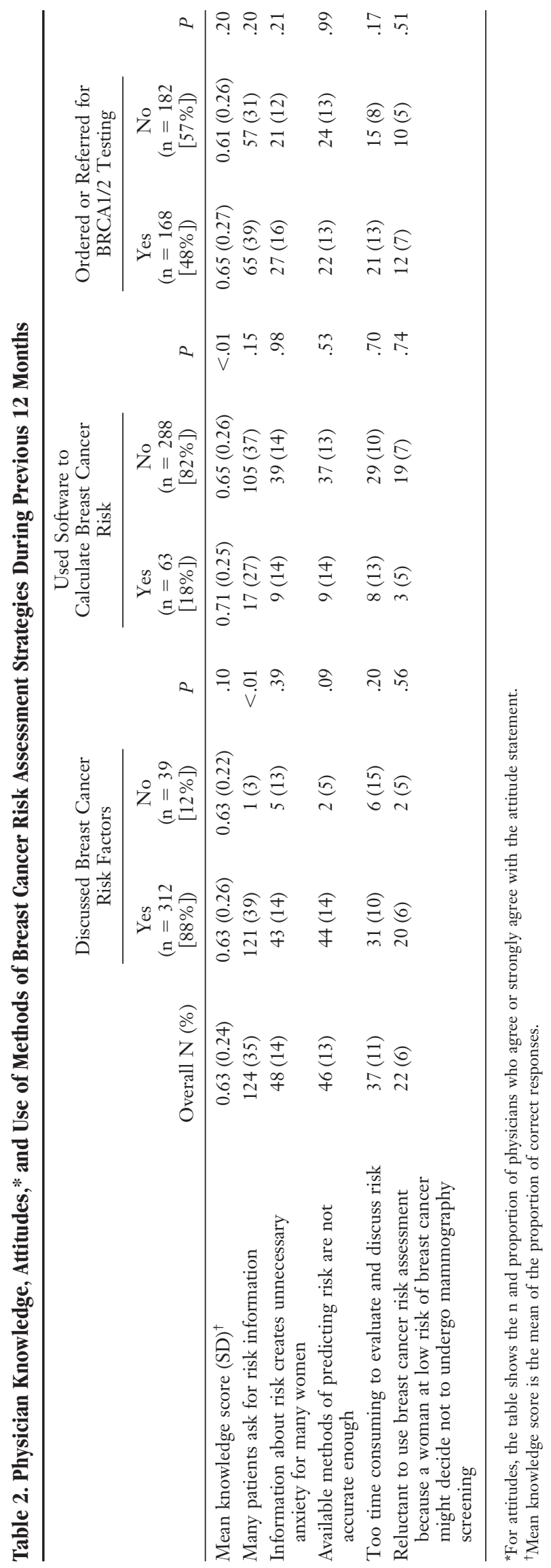


Table 3. Adjusted Association Between Physician Demographics, Attitudes, Knowledge, and Use of Methods of Breast Cancer Risk Assessment*

\begin{tabular}{lccc}
\hline & $\begin{array}{c}\text { Discussed Breast Cancer } \\
\text { Risk Factors }\end{array}$ & $\begin{array}{c}\text { Used Software to Calculate } \\
\text { Breast Cancer Risk }\end{array}$ & $\begin{array}{c}\text { Ordered or Referred for } \\
\text { BRCA1/2 Testing }\end{array}$ \\
\hline Specialty & & & 1.00 \\
$\quad$ Internal medicine (ref) & 1.00 & $0.83(0.37-1.81)$ & $0.95(0.57-1.59)$ \\
$\quad$ Family practice & $1.47(0.58-3.71)$ & $5.37(2.49-11.55)$ & $2.36(1.24-4.49)$ \\
$\quad$ Obstetrics/gynecology & $3.35(1.01-11.13)$ & $0.16(0.02-1.36)$ & $0.27(0.07-0.96)$ \\
Solo practice & $0.14(0.04-0.56)$ & $2.76(1.27-5.30)$ & $1.19(0.59-2.47)$ \\
Family member with breast cancer & $1.63(0.41-6.39)$ & $0.83(0.42-1.61)$ & $1.52(0.95-2.44)$ \\
Attitudes & & & $1.82(0.73-4.55)$ \\
$\quad \begin{array}{l}\text { Many patients ask for information about } \\
\quad \text { their risk of breast cancer }\end{array}$ & $24.60(3.44-195.82)$ & $4.57(1.17-17.08)$ & \\
Knowledge & & & \\
$\quad$ Accuracy score (one point increase) & $1.23(0.26-5.69)$ & & \\
\hline
\end{tabular}

*Models include all the variables in the table as well as physician age, sex, and number of patients seen per week. All data provided as odds ratio $(95 \% \mathrm{CI})$.

many patients asked for risk information was higher among physicians who had discussed breast cancer risk $(P<.01)$ but was not significantly associated with use of software. None of the other attitudes were associated with the use of the breast cancer risk assessment strategies.

The results of the multivariate logistic regression models are shown in Table 3. Physician specialty remained strongly associated with each of the breast cancer risk assessment strategies, with greater odds among obstetrician-gynecologists than internists or family practitioners (for discussion of risk factors: odds ratio [OR], 3.35; 95\% CI, 1.01-11.13; for the use of software: OR, $5.37 ; 95 \%$ CI, 2.54-11.55; and for the use of BRCA1/2 testing: OR, 2.36; 95\% CI, 1.24-4.49). Being in a solo practice was inversely associated with discussion of risk factors and use of $B R C A 1 / 2$ testing use (for discussion of risk factors: OR, 0.14; $95 \%$ CI, 0.040.56; for use of BRCA1/2 testing: OR, 0.27; 95\% CI, 0.07-0.96). There was a trend to an inverse association with the use of risk software but it did not meet statistical significance. In addition, the use of breast cancer risk software was associated with greater knowledge of breast cancer risk factors (OR, 4.57; 95\% CI, 1.17-17.08) and having a family member with breast cancer (OR, 2.49; 95\% CI, 1.27-6.32), wheras discussion of breast cancer risk factors was associated with having patients who asked for information about breast cancer risk (OR, 24.60; 95\% CI, 3.23-188.94).

\section{Discussion}

Primary care physicians play a critical role in the identification of women at high risk for breast cancer and can provide a bridge to interventions that estimate and reduce risk. ${ }^{18,19,22,23}$ The tools and rationale for breast cancer risk assessment in primary care have grown substantially during the last 10 years. However, relatively little is currently known about the practice of breast cancer risk assessment in primary care. This study has several important new findings that have implications for breast cancer control.

First, diffusion of breast cancer risk assessment strategies varies substantially. The great majority of primary care physicians have discussed breast cancer risk with patients during the past year, half have ordered or referred a patient for $B R C A 1 / 2$ testing, and fewer than one-fifth have used software programs to calculate risk. Diffusion studies have shown that the distribution of individuals, based on the time of adoption of an innovation, generally follows a normal bell curve and can be separated into 5 categories: (1) innovators (the first $2.5 \%$ of individuals to adopt an innovation); (2) early adopters (the next $13.5 \%$ ); (3) early majority (the next $34 \%$ ); (4) late majority (the next $34 \%$ ); and (5) laggards (the last $16 \%$ to adopt, if the innovation successfully diffuses through the population). ${ }^{24}$ Based on our data, it seems that use of risk software in women's health remains confined to innovators and early adopters, whereas the use of BRCA1/2 
counseling and testing has diffused to the early majority. Diffusion theory suggests that the probability that these technologies will eventually diffuse throughout primary care physicians depends, in part, on whether the early adopters take on the role of opinion leaders in this area as well as the intrinsic value and characteristics of these technologies and local environmental characteristics. ${ }^{24-28}$

Although diffusion theory can provide some guidance when interpreting these data, it is important to recognize that consideration of $B R C A 1 / 2$ testing is currently recommended for a relatively small proportion of women and that patient preferences are an important determinant of appropriate testing use. As many more physicians discuss risk than refer for genetic testing, it is possible that the relatively lower rate of referral for genetic testing is related to patient decisions after risk discussions. Furthermore, providers may be making trade-offs between the different strategies for risk assessment, preferring to discuss risk factors in the office without incurring additional cost to undertaking the cost and potential complications of genetic testing. Although it is likely that most fulltime primary care providers will have patients in their panels who meet criteria for BRCA1/2 testing, decisions about genetic testing are complex and there is no gold standard for the desired rate of testing among high-risk women.

Second, physicians who have adopted novel breast cancer risk assessment strategies differ in several ways from their peers. Obstetrician-gynecologists seem to have adopted these strategies more rapidly than either internists or family practitioners, perhaps because a greater proportion of their care is related to women's health, making breast cancer more salient in their decision making. Early adopters of risk software are more likely to have a family member with breast cancer, another factor that may increase the salience of breast cancer to the individual physician. This finding echoes an earlier survey of California primary care providers that found physicians were more likely to have referred a woman for genetic counseling if they had more cases of breast cancer in their practice. ${ }^{19}$

The strength of this study is that it sampled a nationally representative sample of primary care physicians. The limitations of this study include the relatively low response rate of $49 \%$, despite multiple mailings and reminders. Although this response rate is close to the average response rate of $54 \%$ (SD, 17\%) found in a 1997 review of physician surveys published in medical journals, nonresponders may have differed from responders in ways that may have influenced our results. ${ }^{29-32}$ This overestimation is likely to be lower for breast cancer risk assessment behaviors because there are no mandates for its performance in primary care practice (in contrast to cancer screening), but we did not have the means to confirm physician practice patterns. Several factors that may have influenced adoption were not included in our survey and their influence on the use of risk assessment could not be determined. In particular, the availability of computers and use of an electronic medical record may be important determinants of the use of software programs to calculate risk. Finally, the measures used in this study were developed specifically for this study and we lack of formal psychometric information for the instrument.

Despite these limitations, this study provides new insights into the use of breast cancer risk assessment by primary care physicians. As tools for breast cancer prevention continue to be developed, the diffusion of breast cancer risk assessment into primary care has gained increasing clinical importance. ${ }^{33}$ This study demonstrates that current adoption seems to be largely related to the personal salience of breast cancer among physicians and practice characteristics, rather than attitudes about the strengths and limitations of current risk assessment methods. Strategies to increase the use of breast cancer risk assessment may need to focus on increasing the salience of breast cancer risk in primary care practice and on developing tools and systems that can support the use of these tools among diverse practice sites. Ongoing research into better methods of breast cancer screening and prevention, as well as the identification of new genetic and environmental risk factors, are likely to change the paradigm of breast cancer risk reduction in primary care. These developments may serve to increase the salience of breast cancer risk assessment to the average primary care provider, but they must also be accompanied by strategies and tools to facilitate their efficient incorporation into primary care practice if they are to reach their full potential in reducing breast cancer mortality. 


\section{References}

1. Jemal A, Siegel R, Ward E, Murray T, Xu J, Thun MJ. Cancer statistics, 2007. CA Cancer J Clin 2007;57:43-66.

2. Ries LAG, Harkins D, Krapcho M, et al. SEER Cancer Statistics Review, 1975-2003. Bethesda (MD): National Cancer Institute, 2006.

3. Gail MH, Brinton LA, Byar DP, et al. Projecting individualized probabilities of developing breast cancer for white females who are being examined annually. J Natl Cancer Inst 1989;81:1879-86.

4. Armstrong K, Eisen A, Weber B. Assessing the risk of breast cancer. N Engl J Med 2000;342:564-71.

5. Fisher B, Costantino JP, Wickerham DL, et al. Tamoxifen for the prevention of breast cancer: current status of the National Surgical Adjuvant Breast and Bowel Project P-1 study. J Natl Cancer Inst 2005;97:1652-62.

6. Freedman AN, Graubard BI, Rao SR, McCaskillStevens W, Ballard-Barbash R, Gail MH. Estimates of the number of US women who could benefit from tamoxifen for breast cancer chemoprevention. J Natl Cancer Inst 2003;95:526-32.

7. Rebbeck TR, Friebel T, Lynch HT, et al. Bilateral prophylactic mastectomy reduces breast cancer risk in BRCA1 and BRCA2 mutation carriers: the PROSE Study Group [see comment]. J Clin Oncol 2004;22:1055-62.

8. Gail MH, Costantino JP. Validating and improving models for projecting the absolute risk of breast cancer [comment]. J Natl Cancer Inst 2001;93:334-5.

9. Shattuck-Eidens D, Oliphant A, McClure M, et al. BRCA1 sequence analysis in women at high risk for susceptibility mutations. Risk factor analysis and implications for genetic testing. JAMA 1997;278:1242-50.

10. Couch F, DeShano M, Blackwood M, Weber B. BRCA1 mutations in women attending clinics that evaluate the risk of breast cancer. $\mathrm{N}$ Engl J Med 1997;336:1409-15.

11. Frank TS, Manley SA, Olopade OI, et al. Sequence analysis of BRCA1 and BRCA2: correlation of mutations with family history and ovarian cancer risk. J Clin Oncol 1998;16:2417-25.

12. Claus EB, Risch N, Thompson WD. Autosomal dominant inheritance of early-onset breast cancer: Implications for risk prediction. Cancer 1994;73:643-51.

13. Parmigiani G, Berry D, Aguilar O. Determining carrier probabilities for breast cancer-susceptibility genes BRCA1 and BRCA2. Am J Hum Genet 1998;62:145-58.

14. Domchek SM, Eisen A, Calzone K, Stopfer J, Blackwood A, Weber BL. Application of breast cancer risk prediction models in clinical practice. J Clin Oncol 2003;21:593-601.

15. Gail M, Rimer B. Risk-based recommendations for mammographic screening for women in their forties [see comment]. J Clin Oncol 1998;16:3105-14.

16. Rossouw JE, Anderson GL, Prentice RL, et al. Risks and benefits of estrogen plus progestin in healthy postmenopausal women: principal results from the
Women's Health Initiative randomized controlled trial. JAMA 2002;288:321-33.

17. US Preventive Services Task Force. Genetic risk assessment and BRCA mutation testing for breast and ovarian cancer susceptibility. Ann Intern Med 2005;143:355-61.

18. Haas J, Kaplan C, Gregorich S, Perez-Stable E, Des Jarlais G. Do physicians tailor their recommendations for breast cancer risk reduction based on patient risk? J Gen Intern Med 2004;19:302-8.

19. Kaplan CP, Haas JS, Perez-Stable EJ, Des Jarlais G, Gregorich SE. Factors affecting breast cancer risk reduction practices among California physicians. Prev Med 2005;41:7-15.

20. Green L, Eriksen M, Schor E. Preventive practices by physicians: behavioral determinants and potential interventions. Am J Prev Med 1988;4:101-7.

21. Green L, Kreuter M. Health promotion planning. 2nd ed. Mountain View: Mayfield Publishing Co; 1991.

22. Armstrong K, Quistberg DA, Micco E, Domchek S, Guerra C. Prescription of tamoxifen for breast cancer prevention by primary care physicians. Arch Intern Med 2006;(166):2260-5.

23. Wideroff L, Freedman AN, Olson L, et al. Physician use of genetic testing for cancer susceptibility: results of a national survey. Cancer Epidemiol Biomarkers Prev 2003;12:295-303.

24. Rogers E. Diffusion of innovations. New York: Free Press; 1995.

25 Greenberg MR. The diffusion of public health innovations. Am J Public Health 2006;96:209-10.

26. Rogers EM. A prospective and retrospective look at the diffusion model. J Health Commun 2004; 9(Suppl 1):13-9.

27. Haider M, Kreps GL. Forty years of diffusion of innovations: utility and value in public health. J Health Commun 2004;9(Suppl 1):3-11.

28. Berwick DM. Disseminating innovations in health care. JAMA 2003;289:1969-75.

29. Asch DA, Jedrziewski MK, Christakis NA. Response rates to mail surveys published in medical journals. J Clin Epidemiol 1997;50:1129-36.

30. Romm FJ, Hulka BS, Kelly LW Jr. Internists' perceptions and performance in office practice. South Med J 1980;73:405-10.

31. McPhee S, Richard R, Solkowitz S. Performance of cancer screening in a university general internal medicine practice: comparison with the 1980 American Cancer Society Guidelines. J Gen Int Med 1986;1:275-81.

32. Wu L, Ashton C. Chart review. A need for reappraisal. Eval Health Prof 1997;20:146-63.

33. Nelson H, Huffman L, Fu R, Harris E, Walker M, Bougatsos C. Genetic risk assessment and BRCA mutation testing for breast and ovarian cancer susceptibility. Portland: Oregon Evidence-based Practice Center, Oregon Health \& Science University; 2005: contract number 290-02-0024. 